\title{
Tratamiento del adulto mayor con síndrome coronario agudo
}

\author{
Carolina Pemberthy-López ${ }^{\mathrm{a}, \mathrm{g}, *}$, César Caraballo-Cordovez ${ }^{\mathrm{b}, \mathrm{g}}$, \\ Simón Gallo-Echeverri ${ }^{\mathrm{b}, \mathrm{g}}$, Nicolás Jaramillo-Gómez ${ }^{\mathrm{c}, \mathrm{g}}$, \\ Camilo Velásquez-Mejía ${ }^{\mathrm{d}, \mathrm{g}}$, Jonathan Cardona-Vélez ${ }^{\mathrm{e}, \mathrm{g}}$ y Heidy Contreras-Martínez ${ }^{\mathrm{f}, \mathrm{g}}$
}

\author{
a Facultad de Medicina Interna, Universidad de Antioquia, Medellín, Antioquia, Colombia \\ b Facultad de Medicina, Universidad de Antioquia, Medellín, Antioquia, Colombia \\ c Universidad CES, Universidad de Córdoba, Medellín, Antioquia, Colombia \\ d Universidad CES, Medellín, Antioquia, Colombia \\ e Universidad Pontificia Bolivariana, Medellín, Antioquia, Colombia \\ f Universidad de Antioquia, Universidad CES, Medellín, Antioquia, Colombia \\ s Grupo de Investigación Cardiovital, CEMDE, Universidad CES, Medellín, Antioquia, Colombia
}

Recibido el 22 de diciembre de 2015; aceptado el 25 de abril de 2016

Disponible en Internet el 7 de julio de 2016

\section{PALABRAS CLAVE \\ Síndrome coronario agudo; Anciano; \\ Edad}

\begin{abstract}
Resumen El adulto mayor se encuentra en una etapa de la vida con cambios fisiológicos importantes que posee repercusiones directas e indirectas sobre su estado clínico, es así como el enfoque diagnóstico y terapéutico de sus patologías debe ser abordado desde una perspectiva diferente, con consideraciones especiales para este grupo poblacional. Lamentablemente, este grupo no ha sido bien representado en los diferentes estudios y por tanto, es mucho lo que desconocemos; su comportamiento y la eficacia de las distintas terapias no están bien establecidos. Teniendo de referencia el síndrome coronario agudo como principal causa de mortalidad en pacientes mayores de 65 años de edad, abordaremos esta entidad desde la evidencia que nos aportan algunos de los registros más importantes al respecto en adultos mayores.

(c) 2016 Sociedad Colombiana de Cardiología y Cirugía Cardiovascular. Publicado por Elsevier España, S.L.U. Este es un artículo Open Access bajo la licencia CC BY-NC-ND (http:// creativecommons.org/licenses/by-nc-nd/4.0/).
\end{abstract}

\section{Treatment of older adults with acute coronary syndrome}

\begin{abstract}
Older adults find themselves at a stage in their life with important physiological alterations that have a direct and indirect impact on their clinical status, hence why the diagnostic and therapeutic approach of their conditions must be faced from a different perspective, taking into account specific considerations for this population group. Unfortunately, this group has not
\end{abstract}

\footnotetext{
* Autor para correspondencia.

Correo electrónico: caropember@gmail.com (C. Pemberthy-López). 
received proper representation in different studies and therefore a lot remains unknown: their behaviour and efficacy of various therapies have not been appropriately established. Taking into account acute coronary syndrome as the main cause for mortality in patients over 65 years old, we will face this condition from evidence gathered from some of the most important registers regarding this topic in older adults.

(c) 2016 Sociedad Colombiana de Cardiología y Cirugía Cardiovascular. Published by Elsevier España, S.L.U. This is an open access article under the CC BY-NC-ND license (http:// creativecommons.org/licenses/by-nc-nd/4.0/).

\section{Introducción}

La enfermedad cardiaca isquémica es la principal causa de muerte en la población mundial ${ }^{1}$. El síndrome coronario agudo (SCA) es la manifestación clínica más frecuente y una de las más retadoras de la enfermedad isquémica coronaria, como tal, tiene una gran prevalencia en la población mayor de 65 años de edad ${ }^{2}$, dadas las condiciones de esta población en cuanto al proceso de envejecimiento del sistema endotelial, en general, el cual afecta todo el sistema cardiovascular. Lo anterior explica que la cardiopatía isquémica sea la primera causa de muerte en mayores de 65 años de edad ${ }^{3}$.

A medida que las condiciones de vida mejoran y los avances científicos en el área médica facilitan la prolongación de la vida, la población de adultos mayores aumenta de forma significativa, incrementado así las patologías complejas y mórbidas como el SCA. Es así como en los registros europeos, los pacientes mayores de 75 años de edad, representan entre el 27 y el $34 \%$ de todos los SCA ${ }^{4}$ y en los Estados Unidos los mayores de 65 años de edad, corresponden al $83 \%$ de todas las muertes por enfermedad cardiaca isquémica, además, en el año 2004 el SCA fue responsable del 35\% de todas las muertes en mayores de 65 años de edad ${ }^{1}$. En Inglaterra la incidencia de infarto agudo de miocardio es de 53 por cada 100.000 habitantes para la población entre 65 y 74 años de edad, siendo esta cifra mucho más alta en la población mayor de 85 años de edad, en la cual asciende a 199 por cada $100.000^{5}$.

En Colombia, al igual que las tendencias mundiales, la población de adultos mayores ha venido creciendo de una forma muy representativa debido a que la expectativa de vida ha pasado de estar estimada en 67,8 años de edad, para el período comprendido entre los años 1985 y 1990, a 73,9 años de edad, para el período 2010-2015, lo que ha hecho que la pirámide poblacional cambie de conformación. Esta tendencia se evidencia en las estadísticas reportadas por el Departamento Administrativo Nacional de Estadística (DANE), que posiciona a la enfermedad cardiaca isquémica como la principal causa de muerte en personas mayores de 55 años de edad, lo cual representa uno de los mayores retos que tiene que enfrentar el sistema de salud en la actualidad $^{6}$.

Por razones no bien establecidas, los estudios y registros a nivel mundial han excluido de forma sistemática la población de adultos mayores, creando así una brecha en el conocimiento basado en la evidencia científica para el tratamiento de la enfermedad cardiaca isquémica en el adulto mayor.
Las actuales guías, por las cuales se rigen los diferentes servicios de dolor torácico y urgencias, necesitan claridad y datos seguros sobre los adecuados algoritmos, indicaciones y contraindicaciones de los procedimientos para los SCA en estos pacientes teniendo en cuenta que sus condiciones de base los dejan expuestos a un mayor riesgo de desenlaces adversos $^{7}$. Por lo anterior se hace crucial incluir esta población en los registros y estudios prospectivos aleatorizados de enfermedades isquémicas coronarias, para así lograr establecer el verdadero riesgo y los desenlaces adversos en este grupo considerado el más vulnerable.

\section{Características clínicas}

A pesar de que la molestia torácica en el SCA sigue siendo el síntoma más común ${ }^{8,9}$, en los adultos mayores es menos frecuente, dándole cabida a otros síntomas atípicos. Esto junto a una mayor incidencia de enfermedades con presentaciones clínicas similares, genera un reto clínico para el diagnóstico ${ }^{3}$. Es muy importante, para el médico conocer la frecuencia de los tipos de presentación con el fin de identificar, clasificar y proporcionar un tratamiento oportuno a estos pacientes, previniendo así complicaciones a corto y largo plazo.

Existen factores que aumentan la presentación atípica del SCA como la historia de: la hipertensión arterial, la diabetes y la falla cardiaca. Estos a su vez son más frecuentes en los adultos mayores ${ }^{10}$. En la tabla 1 se enumeran los signos y síntomas más frecuentes en la presentación clínica del SCA en la población de los adultos mayores.

En los mayores de 85 años de edad, el dolor torácico se presenta solo en el $40 \%$ y hasta el $40 \%$ presentan cambios electrocardiográficos atípicos ${ }^{5}$, mientras que un $60 \%$ presentan infartos silentes ${ }^{1}$. Otros hallazgos atípicos en la presentación del SCA en esta población son: la fatiga, la astenia, el edema agudo de pulmón, el accidente cerebrovascular, las arritmias y la muerte súbita ${ }^{8}$.

\section{Tratamiento}

Las guías actuales para el manejo del infarto agudo de miocardio con y sin elevación del ST plantean que el tratamiento efectivo en la población más joven, usualmente también está indicado en los pacientes de edad avanzada, dejando como recomendación, por la falta de evidencia, individualizar la toma de decisiones según las características 
Tabla 1 Presentación clínica

\begin{tabular}{|c|c|c|}
\hline Estudio & Población & Resultados \\
\hline Brieger et al. ${ }^{10}$ & $\begin{array}{l}1763 \text { pacientes mayores de } 18 \text { años con SCA sin } \\
\text { dolor torácico, de los cuales el } 70,6 \% \text { fue } \\
\text { mayor de } 65 \text { años }\end{array}$ & $\begin{array}{l}\text { - Disnea } 49,3 \% \\
\text { - Diaforesis } 26,2 \% \\
\text { - Náuseas o vómito } 24,3 \% \\
\text { - Síncope/presíncope } 19,1 \%\end{array}$ \\
\hline Grosmaitre et al. ${ }^{28}$ & $\begin{array}{l}255 \text { pacientes con infarto agudo de miocardio } \\
\text { con elevación del ST (IAMCEST) } \geq 75 \text { años }\end{array}$ & $\begin{array}{l}\text { - Dolor torácico } 41,2 \% \\
\text { - Desmayo y/o caída } 15,7 \% \\
\text { - Disnea } 15,7 \% \\
\text { - Síntomas gastrointestinales } 9,8 \% \\
\text { - Deterioro del estado general } 6,7 \% \\
\text { - Delirium } 5 \%\end{array}$ \\
\hline
\end{tabular}

del paciente como sus preferencias, comorbilidades, estado funcional y cognitivo, y expectativa de vida ${ }^{11,12}$.

\section{Manejo farmacológico}

Un análisis del estudio CRUSADE mostró que a mayor edad las contraindicaciones y el menor uso de medicamentos fundamentales en los estados isquémicos (el ácido acetilsalicílico, los betabloqueadores y la heparina), se reportaban con mayor frecuencia. La edad tuvo su mayor impacto en el uso de los antiagregantes plaquetarios como: el clopidogrel y los inhibidores de las glicoproteínas IIb/IIla, pues a pesar de que el $92 \%$ de los mayores de 85 años de edad, tenían biomarcadores de daño miocárdico positivos, solo el $30 \%$ recibieron el clopidogrel y $12,8 \%$ los inhibidores de las glicoproteínas $\mathrm{Ilb} / \mathrm{Illa}^{13}$. Otros estudios realizados entre los años 2003 y el 2010, muestran una tendencia diferente en este grupo etario, evidenciándose un uso más adecuado de la terapia farmacológica ${ }^{14}$. Un estudio observacional con 13.662 pacientes mayores de 70 años de edad, con SCA, evidenció que el incremento en el uso de los medicamentos recomendados se relacionó con una disminución significativa en mortalidad y desenlaces adversos cardiovasculares ${ }^{15}$.

La evidencia científica apunta a que los adultos mayores deben recibir tratamiento farmacológico similar a los pacientes de menor edad, ajustando a nivel individual por el estado y las comorbilidades. La tabla 2 muestra la evidencia para cada familia de medicamentos sobre la terapia farmacológica en estos pacientes, estas consideraciones se deben tener en cuenta al momento de elegir el enfoque terapéutico del SCA para esta población.

\section{Revascularización}

Debido al crecimiento de la población adulta con una alta prevalencia de la enfermedad coronaria, el tratamiento de la revascularización en esta población debe responder dos preguntas: 1. ¿Es pertinente el tratamiento invasivo frente a un posible tratamiento médico conservador? 2. ¿Cuál es el tratamiento de revascularización más adecuado, quirúrgico o la intervención percutánea coronaria?

La evidencia científica demuestra que en el SCA el tratamiento agresivo (revascularización quirúrgica y percutánea), representa un mayor beneficio comparado con el manejo médico ${ }^{12,13,15}$. El estudio FRISC ॥, en el cual se comparó la terapia inicial conservadora frente a una terapia invasiva dentro de los 7 primeros días en pacientes con síndrome coronario agudo sin elevación del ST, demostró a los 6 meses una tasa menor de muerte o MI, con un beneficio sostenido a los 5 años en pacientes con 65-75 años de edad. A su vez, en el estudio TACTIC TIMI, la terapia invasiva temprana en comparación con la terapia invasiva conservadora demostró un beneficio frente a: la mortalidad, el infarto de miocardio y la rehospitalización por $\mathrm{SCA}^{4}$, (tabla 3 ).

El beneficio de la terapia invasiva no solo impacta en el SCA, ya que la revascularización ha demostrado ser un método adecuado en pacientes ancianos con enfermedad cardiaca isquémica estable, en donde estudios como el TIME y el APPROACH demostraron una mejoría sintomática, en la calidad de vida y una reducción en la muerte, el infarto de miocardio y la readmisión por SCA a los 6 meses y a los 4 años, respectivamente 4 .

Con respecto al manejo percutáneo, Luca y Cols., demuestran en su estudio ${ }^{16}$, que a pesar de una mayor mortalidad a largo plazo comparada con pacientes jóvenes $(H R=2,17$ [IC 95\%, 1,97-2,39]), la edad no tuvo impacto en el riesgo de reinfarto $(p=0,36)$, trombosis del stent $(p=0,84)$ ni revascularización del vaso culpable $(p=0,54)^{16,17}$. Por su parte, Fox y Cols. ${ }^{18}$, evaluaron el impacto de realizar la revascularización invasiva rutinaria (IR) vs. selectiva (IS) en 5.467 pacientes con IAMNST de los cuales el $48,6 \%$ eran $\geq 65$ años, encontrando que aquellos llevados a IR presentaron menor riesgo de muerte cardiovascular o IAM no fatal (HR 0,81; IC 95\%, 0,71-0,93; $p=0,002$ ), menor mortalidad por todas las causas (HR 0,85; IC 95\%, $0,75-0,96 ; p=0,008$ ) y menos IAM no fatal (HR 0,77, IC 95\%, 0,65-0,90; $p=0,001$ ), (tabla 3).

Completando la evidencia en poblaciones de edad avanzada está el estudio «After eighty» realizado en pacientes con diagnóstico de IAMNST o angina inestable y edad $\geq 80$ años de edad, demostró que la estrategia invasiva, incluyendo la $\mathrm{PCl}$ y el bypass coronario más la terapia médica óptima es superior al tratamiento médico óptimo solo, en pacientes clínicamente estables, sin diferencias en las tasas de complicaciones entre ambos grupos. No obstante, no es posible concluir si estos resultados son iguales para aquellos pacientes mayores a 90 años de edad, pues la eficacia de dicha intervención fue menor al aumentar la edad ${ }^{19}$.

Al evaluar las dos opciones de revascularización en la enfermedad isquémica cardiaca (CABG vs. $\mathrm{PCI}$ ) factores como: las morbilidades asociadas, el riesgo de mortalidad 
Tabla 2 Manejo farmacológico

\begin{tabular}{|c|c|}
\hline Medicamento & Evidencia \\
\hline Anticoagulantes & $\begin{array}{l}\text { - Fondaparinux es de elección, pues se asocia con menor riesgo de sangrado y menor mortalidad } \\
\text { a largo plazo. No se recomienda en IAMCEST llevado a intervención coronaria percutánea }(\mathrm{PCl} \text {, } \\
\text { por sus siglas en inglés) primaria }{ }^{17,29} \text {. } \\
\text { - Enoxaparina: no usar bolo en mayores de } 75 \mathrm{años}^{30} \text {. Uso según tasa de filtración glomerular }{ }^{17} \text {. } \\
\text { En ensayos clínicos se ha administrado a dosis de } 0,75 \mathrm{mg} / \mathrm{kg} \text { cada } 12 \text { horas en mayores de } 75 \\
\text { años, en vez de } 1 \mathrm{mg} / \mathrm{kg} \text { cada } 12 \text { horas usado en la población más joven }{ }^{31} \text {. } \\
\text { - La heparina no fraccionada es el medicamento protagonista en el tratamiento de pacientes con } \\
\left.\text { enfermedad renal crónica avanzada (TFG }<30 \mathrm{ml} / \mathrm{min} / 1,73 \mathrm{~m}^{2}\right)^{17} \text {. Pacientes mayores de } 60 \text { años } \\
\text { pueden requerir menores dosis }{ }^{30} \text {. }\end{array}$ \\
\hline Inhibidores G IIb/IIla & $\begin{array}{l}\text { - Eptifibatide es el agente preferido por el beneficio observado. Reducir dosis de infusión de } \\
\text { eptifibatide y tirofiban de acuerdo a la función renal. El aumento de la edad puede aumentar el } \\
\text { riesgo de sangrado }{ }^{30} \text {. } \\
\text { - En general, no hay interacciones de tratamiento relacionado con la edad, aunque en el sexo } \\
\text { femenino se presentaron mayores eventos adversos }{ }^{12} \text {. }\end{array}$ \\
\hline $\begin{array}{l}\text { Antagonistas de } \\
\text { receptores } P 2 Y_{12}\end{array}$ & $\begin{array}{l}\text { - Se sugiere utilizar ticagrelor como medicamento de elección, seguido por clopidogrel como } \\
\text { segunda opción }{ }^{12} \text {. En cuanto a este, aunque la evidencia no es concluyente, se sugiere no } \\
\text { administrar dosis de carga en mayores de } 75 \text { años debido a que puede aumentar el riesgo de } \\
\text { sangrados mayores }{ }^{32-35} \text {. } \\
\text { - Prasugrel: la Food and Drug Administration (FDA) emitió mensaje de alerta por exceso de } \\
\text { sangrado sin beneficio clínico. No se recomienda en } \geq 75 \text { años o }<60 \mathrm{~kg} \text { o pacientes con historia } \\
\text { de accidente cerebrovascular o isquemia cerebral transitoria }{ }^{36} \text {, excepto en aquellos de alto } \\
\text { riesgo como diabéticos o con infarto previo }{ }^{37} \text {. }\end{array}$ \\
\hline Fibrinolíticos & $\begin{array}{l}\text { - Son eficaces en los pacientes de mayor edad; en pacientes } \geq 75 \text { años sometidos a esta terapia, } \\
\text { hay una reducción de la mortalidad relativa del } 15 \%{ }^{1} \text {. } \\
\text { - Como efecto adverso grave, se presenta hemorragia intracraneal en } 2,9 \% \text { en }>85 \text { años }^{1} \text {. } \\
\text { El tenecteplasa se asoció a una frecuencia de hemorragia intracraneal menor que con el activador } \\
\text { de plasminógeno tisular, aunque ninguno mostró superioridad en los resultados cardiacos }{ }^{1} \text {. } \\
\text { - Se deben usar, preferiblemente, con anticoagulantes como enoxaparina pues se ha demostrado } \\
\text { que en }>75 \text { años disminuye el riesgo de sangrado con una dosis de } 0,75 \mathrm{mg} / \mathrm{kg} \text { cada } 12 \text { horas } \\
\text { omitiendo la dosis de carga }{ }^{38} \text {. }\end{array}$ \\
\hline
\end{tabular}

y las posibles complicaciones deben ser tenidas en cuenta. La elección de la estrategia de la revascularización se torna más complicada en los pacientes con la enfermedad multivaso o las afecciones del tronco principal izquierdo, los hallazgos prevalentes en las poblaciones mayores que a su vez presentan altas tasas de comorbilidades. La revascularización quirúrgica presenta mejores resultados a largo plazo ya que se asocia a menos reinfartos, reintervenciones y mayor supervivencia, (tabla 4$)^{20-22}$. No obstante, a corto plazo tiene mayor mortalidad intrahospitalaria comparada con la revascularización percutánea ${ }^{21}$. Weintraub y Cols., demostraron que la mortalidad al año es similar en pacientes revascularizados con $\mathrm{CABG}$ y $\mathrm{PCl}$, no obstante, la CABG presenta una mayor sobrevida y reducción en Ictus e infartos de miocardio a los 4 años de la intervención.

Las guías AHA/ACC establecen que es razonable escoger CABG sobre $\mathrm{PCl}$ en el síndrome coronario agudo sin elevación del ST, particularmente, en pacientes diabéticos o en aquellos con enfermedad triple vaso compleja para reducir: los eventos cardiovasculares, la readmisión y mejorar la sobrevida. Las guías ESC establecen que los ancianos deben ser considerados para una terapia invasiva temprana con la opción de revascularización, después de evaluar los riesgos y los beneficios.

A pesar de que la evidencia indica que los pacientes de edad avanzada obtienen la máxima reducción del riesgo con manejo invasivo y atención integral. Se tienden a omitir tratamientos intervencionistas al igual que a evitar medicamentos para la prevención secundaria con los cuales se podrían beneficiar esta población ${ }^{23}$, además, persiste la discrepancia entre las edades respecto al acceso a un manejo óptimo en los diferentes sistemas de atención en salud, implicando un peor pronóstico para estos pacientes, como se describe en la tabla 4.

Toda la evidencia en estudios clínicos hasta el momento establece que el manejo de poblaciones de pacientes mayores con SCA sin elevación del ST se debe centrar en el paciente específico, considerando sus preferencias y teniendo en cuenta: las comorbilidades, el estado funcional y cognitivo al igual que su expectativa de vida.

\section{Fragilidad}

Debido a la mayor proporción de población anciana e incremento de la complejidad de los pacientes, la fragilidad se ha convertido en un aspecto sobresaliente en la medicina cardiovascular. Constituye un síndrome biológico que refleja un estado de menor reserva fisiológica y vulnerabilidad ante estresores (por ejemplo: enfermedades agudas o crónicas,

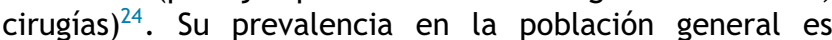
de $10 \%$ y en personas con enfermedad cardiovascular 
Tabla 3 Revascularización

\begin{tabular}{|c|c|c|c|}
\hline $\begin{array}{l}\text { Autores y tipo de } \\
\text { estudio }\end{array}$ & Población & Desenlaces principales & Resultados \\
\hline \multicolumn{4}{|c|}{ Manejo invasivo vs. manejo médico } \\
\hline Graham MM et al. ${ }^{39}$ & $\begin{array}{l}6.181 \text { pacientes }>70 \\
\text { años }\end{array}$ & $\begin{array}{l}\text { Mortalidad a uno y cuatro } \\
\text { años }\end{array}$ & $\begin{array}{l}\text { RAR en mortalidad a } 4 \text { años en } \geq 80 \text { años } \\
\text { de } 17 \% \text { y } 11,3 \% \text { para revascularización } \\
\text { quirúrgica y percutánea vs. terapia } \\
\text { médica respectivamente }{ }^{38} \text {. }\end{array}$ \\
\hline Zaman et al. ${ }^{23}$ & $\begin{array}{l}155.818 \text { pacientes } \\
\text { con IAM con } \\
95,824>65 \text { años }\end{array}$ & $\begin{array}{l}\text { Tendencia de tratamiento por } \\
\text { grupo etario y supervivencia }\end{array}$ & $\begin{array}{l}\text { Menor tendencia a hacer manejo } \\
\text { intensivo y a hacer prevención } \\
\text { secundaria en los pacientes más viejos. } \\
\text { Mayor supervivencia ( } 2,29 \text { años) con } \\
\text { manejo intensivo en todas las edades } \\
\text { para IAMST e IAMNST; este beneficio fue } \\
\text { menor al aumentar la edad. }\end{array}$ \\
\hline Tegn et al. ${ }^{44}$ & $\begin{array}{l}457 \text { pacientes } \geq 80 \\
\text { años con } \\
\text { IAMNST/Angina } \\
\text { inestable }\end{array}$ & $\begin{array}{l}\text { IAM, revascularización } \\
\text { urgente, ACV y muerte }\end{array}$ & $\begin{array}{l}\text { Los desenlaces ocurrieron en el } 40,6 \% \text { de } \\
\text { los pacientes sometidos al grupo de } \\
\text { intervención y en el } 61,4 \% \text { de aquellos } \\
\text { con manejo conservador. HR } 0,53 \text { (IC } \\
95 \%, 0,41-0,69 \text { ). P }=0,0001 \text {; este } \\
\text { beneficio fue menor al aumentar la edad } \\
\text { No hubo diferencias en las tasas de } \\
\text { sangrado entre ambos grupos }\end{array}$ \\
\hline \multicolumn{4}{|c|}{ Revascularización percutánea vs. trombólisis } \\
\hline Grines $C$ et al. ${ }^{40}$ & $\begin{array}{l}2.275 \text { pacientes con } \\
\text { IAMST, con } 805>70 \\
\text { años }\end{array}$ & Mortalidad a 30 días & $\begin{array}{l}\text { En }>70 \text { años, mortalidad de } 13,3 \% \text { vs. } \\
23,6 \% \text { con revascularización percutánea } \\
\text { vs. trombólisis; } p<0,05 ; \text { NNT }=8^{3,40}\end{array}$ \\
\hline \multicolumn{4}{|c|}{ Revascularización quirúrgica vs. percutánea } \\
\hline $\begin{array}{l}\text { Hlatky et al. } \\
\text { Metanálisis }\end{array}$ & $\begin{array}{l}7.812 \text { pacientes con } \\
\text { enfermedad } \\
\text { coronaria de varios } \\
\text { vasos }\end{array}$ & Mortalidad a 5 años & $\begin{array}{l}\text { No diferencias significativas de } \\
\text { mortalidad en los grupos de tratamiento. } \\
\text { Menor mortalidad con revascularización } \\
\text { quirúrgica en los pacientes diabéticos } \\
\text { (HR } 0,70 \text {, IC } 0,56-0,87 \text { ) y en } \geq 65 \text { años } \\
\text { (HR } 0,82 \text {, IC } 0,70-0,97 \mathrm{p}=0,002 \text { ). Los } \\
\text { desenlaces (nueva revascularización y } \\
\text { muerte, IAM o nueva revascularización) } \\
\text { fueron menores con revascularización } \\
\text { quirúrgica. No hay según el número de } \\
\text { vasos comprometidos. }\end{array}$ \\
\hline Dacey et al. ${ }^{21}$ & $\begin{array}{l}1.693 \text { pacientes entre } \\
80-89 \text { años. Sin } \\
\text { enfermedad de } \\
\text { arteria coronaria } \\
\text { izquierda o historia } \\
\text { de revascularización. }\end{array}$ & $\begin{array}{l}\text { Mortalidad intrahospitalaria, } \\
\text { a } 6 \text { meses y } 8 \text { años }\end{array}$ & $\begin{array}{l}\text { Con revascularización quirúrgica hubo } \\
\text { mayor mortalidad intrahospitalaria }(5,9 \% \\
\text { vs. } 3 \% ; p=0,005) \text { y en los primeros } 6 \\
\text { meses (HR: } 1,32 ; \text { IC } 0,92-1,90 \text {, } \\
p=0,135) \text {. Además, mayor supervivencia } \\
\text { entre } 6 \text { meses y } 8 \text { años (HR } 0,72 ; \text { IC } \\
0,57-0,91, p=0,005) \text {. }\end{array}$ \\
\hline Nicolini et al. ${ }^{22}$ & $\begin{array}{l}\text { 1.388 pacientes entre } \\
80-89 \text { años }\end{array}$ & $\begin{array}{l}\text { Mortalidad a } 30 \text { días y } 7 \text { años, } \\
\text { muerte de origen cardiaco, } \\
\text { IAM, nueva revascularización } \\
\text { y ACV a } 7 \text { años. }\end{array}$ & $\begin{array}{l}\text { No hay diferencia significativa en } \\
\text { mortalidad a } 30 \text { días. A } 7 \text { años la PCI } \\
\text { tuvo mayor muerte de origen cardiaca, } \\
\text { IAM y nueva revascularización. Sin } \\
\text { diferencias en los grupos en cuanto al } \\
\text { ACV. La PCI fue un predictor } \\
\text { independiente de muerte a } 7 \text { años. }\end{array}$ \\
\hline
\end{tabular}

oscila entre 10-60\%, incrementando la mortalidad 2 veces $^{24,25}$. La causa de esta declinación funcional es multifactorial e involucra múltiples órganos y sistemas ${ }^{25}$. Cuando un paciente frágil se expone a algún estresor está en riesgo de presentar: una descompesación marcada y desproporcionada, eventos adversos, complicaciones de procedimientos, recuperación prolongada, declinación funcional, discapacidad y mortalidad ${ }^{24}$. 
Tabla 4 Estrategias de manejo de síndrome coronario agudo en edad avanzada

\begin{tabular}{|c|c|}
\hline Estrategia & Evidencia \\
\hline Oportunidad de la atención & $\begin{array}{l}\text { Los adultos mayores presentan los tiempos puerta-electrocardiograma, } \\
\text { puerta-balón y puerta-aguja más prolongados }{ }^{41} \text {. }\end{array}$ \\
\hline Revascularización & $\begin{array}{l}\text { En pacientes ancianos elegibles para el procedimiento y sin } \\
\text { contraindicaciones, la revascularización es menos realizada }{ }^{41} \text {. } \\
\text { Entre } 2006-2010 \text { de los pacientes con IAMST se revascularizó } 55 \% \text { de los }>85 \\
\text { años contra } 84 \% \text { entre } 18-65 \text { años. En IAMNST se revascularizó el } 14 \% \text { de } \\
\text { los }>85 \text { años contra } 83 \% \text { de aquellos entre } 18-65 \text { años }^{23} \text {. }\end{array}$ \\
\hline Manejo en unidades especializadas & $\begin{array}{l}\text { Durante la estancia hospitalaria, los pacientes } \geq 65 \text { años tienen menor } \\
\text { probabilidad de ser admitidos a la unidad de cuidado coronario (Ingresa } 64 \% \\
\text { de los }<65 \text { años, } 52,9 \% \text { entre } 65-84 \text { años y } 39,1 \% \text { en } \geq 85 \text { años) }\end{array}$ \\
\hline Atención por cardiología & $\begin{array}{l}\text { Durante la estancia hospitalaria los pacientes } \geq 65 \text { años tienen menor } \\
\text { probabilidad de estar bajo el cuidado de cardiología }(51,1 \% \text { de los }<65 \text { años, } \\
42,2 \% \text { entre } 65-84 \text { años y } 30,1 \% \text { en } \geq 85 \text { años })^{42} \text {. }\end{array}$ \\
\hline Medicamentos para prevención secundaria & $\begin{array}{l}\text { Entre } 2003-2010 \text { se encontró un incremento en el uso de aspirina }(93,7 \% \text { a } \\
98,4 \%) \text {, Clopidogrel }(47,2 \% \text { a } 95,7 \% \text {, Beta bloqueadores }(87 \% \text { a } 95,7 \%), \\
\text { inhibidores de la enzima convertidora de angiotensina }(82,6 \% \text { a } 94,4 \%) \\
\text { estatinas }(88,3 \% \text { a } 96,5 \%)^{42} \text {. } \\
\text { Sin embargo, la probabilidad de recibir estos medicamentos aun disminuye } \\
\text { con la edad }{ }^{14,23,42} \text {. }\end{array}$ \\
\hline Rehabilitación cardiaca & $\begin{array}{l}\text { El acceso es pobre, solo el } 13,9 \% \text { de los pacientes con infarto agudo de } \\
\text { miocardio y } 31 \% \text { de los sometidos a revascularización quirúrgica }>65 \text { años } \\
\text { participan en programas de rehabilitación cardiaca y la cantidad disminuye a } \\
\text { mayor edad. De los mayores de } 85 \text { años, solo el } 4,6 \% \text { asiste a estos programas } \\
\text { posteriores al evento coronario comparado con } 26,6 \% \text { de aquellos entre } 65-74 \\
\text { años }^{5} \text {. }\end{array}$ \\
\hline Educación & $\begin{array}{l}\text { Ser mayor de } 70 \text { años está asociado con menor probabilidad de recibir } \\
\text { recomendaciones sobre hábitos de vida saludables }{ }^{43} \text {. }\end{array}$ \\
\hline
\end{tabular}

Las innovaciones técnicas de las que se dispone actualmente permiten usar una serie de dispositivos y procedimientos, sin embargo, surge la duda sobre el beneficio individual en ciertos pacientes sumado a los costos en los sistemas de salud, lo que obliga a intensificar la selección de los mismos. De esta forma se busca prevenir que un paciente reciba una intervención costosa pero fútil ${ }^{24}$. Ante ello, evaluar la fragilidad permite estimar el riesgo y guiar un manejo personalizado que maximice la probabilidad de un desenlace positivo, teniendo en cuenta que el paciente frágil enfrenta un mayor riesgo derivado de procedimientos invasivos pero también un beneficio potencial de intervenciones como la rehabilitación cardiaca.

Se han desarrollado más de 20 herramientas para evaluar la fragilidad, lo que explica la variabilidad en los estudios y la confusión respecto a cuál herramienta usar. La mayoría se basa en 1 o más de los 5 dominios centrales que define el fenotipo: la lentitud, la debilidad, la baja actividad física, el agotamiento y el desgaste ${ }^{24-26}$; estos se pueden considerar individualmente o en varias escalas, ver tabla 5.

La discapacidad, definida como la dificultad o dependencia para desempeñar actividades de la vida diaria o actividades instrumentales, erróneamente se considera como la fragilidad. No obstante, es más un desenlace adverso asociado con la fragilidad o una entidad separada pero no intercambiable a la hora de evaluar los pacientes ${ }^{24}$.
Respecto a la enfermedad coronaria, un estudio en ancianos llevados a $\mathrm{PCl}$ en la Clínica Mayo, la fragilidad aumentó el riesgo de mortalidad a 3 años (28\% vs. $6 \%$; OR 2,74). Otro estudio en pacientes llevados a $\mathrm{PCl}$ encontró la «caquexia/fragilidad» como el predictor más potente de la mortalidad a 18 meses (HR: 14). Igualmente, un estudio en $>75$ años con IAMNST concluyó que la fragilidad tenía una asociación independiente con la mortalidad intrahospitalaria, la mortalidad a un mes y cuidados hospitalarios prolongados; en el seguimiento a un año hubo un incremento en la mortalidad en pacientes frágiles comparado con los no frágiles (HR 4,3, IC 95\% $2,4-7,8)^{27}$.

Varios estudios han demostrado que los pacientes frágiles fueron manejados de forma menos agresiva comparada con aquellos no frágiles, sin embargo, aun no es claro si esto es beneficiosos o no para este grupo poblacional. Además, era menos probable que recibieran IECA ( $71 \%$ vs. $81 \%$ y betabloqueadores (63\% vs. $80 \%$ ), tenían menos probabilidad de admisión a la unidad coronaria (35\% vs. $54 \%)$, realización de $\mathrm{PCl}(15 \%$ vs. $46 \%)$ o CABG ( $9 \%$ vs. $16 \%)^{24}$.

Lo anterior muestra la importancia de evaluar la fragilidad en la valoración integral del paciente de edad avanzada y la toma de decisiones en su manejo. Para ello se requiere el establecimiento de una escala de fragilidad de fácil aplicación y su inclusión en escalas de predicción de morbilidad y mortalidad asociada con intervenciones cardiovasculares. 
Tabla 5 Evaluación de la fragilidad

\begin{tabular}{|c|c|c|c|}
\hline Dominio & Herramienta & Definición & Puntos de corte \\
\hline Lentitud & $\begin{array}{l}\text { Prueba de velocidad } \\
\text { de la marcha en } 5 \mathrm{~m}\end{array}$ & $\begin{array}{l}\text { Paciente detrás de la línea de } \\
\text { inicio, se le solicita que camine a } \\
\text { un ritmo cómodo hasta pasar la } \\
\text { línea final a los } 5 \mathrm{~m} \text {. Repetir } 3 \text { veces } \\
\text { y promediar. }\end{array}$ & $\begin{array}{l}\text { Lento: }<0,83 \mathrm{~m} / \mathrm{s}(<6 \mathrm{~s}) \\
\text { Muy lento: }<0,65 \mathrm{~m} / \mathrm{s}(>7,7 \mathrm{~s}) \\
\text { Extremadamente lento: }<0,50 \mathrm{~m} / \mathrm{s} \\
(>10 \mathrm{~s})\end{array}$ \\
\hline Debilidad & $\begin{array}{l}\text { Prueba de fuerza de } \\
\text { tensión }\end{array}$ & $\begin{array}{l}\text { Se le pide al paciente que apriete } \\
\text { un dinamómetro lo más fuerte } \\
\text { posible, repetir } 3 \text { veces (una con } \\
\text { cada mano y otra con la más } \\
\text { fuerte), reportar el valor máximo. }\end{array}$ & $\begin{array}{l}\text { Hombre: }<30 \mathrm{~kg} . \\
\text { Mujer: }<20 \mathrm{~kg} .\end{array}$ \\
\hline Baja actividad física & $\begin{array}{l}\text { Cuestionarios de } \\
\text { actividad física }\end{array}$ & $\begin{array}{l}\text { Existen varios cuestionarios. Se } \\
\text { recomienda los que miden actividad } \\
\text { en Kcal/semana (Minnesota Leisure } \\
\text { Time Activity, PASE, Paffenbarger } \\
\text { Physical Activity Questionnaire) }\end{array}$ & $\begin{array}{l}\text { Hombre: }<383 \mathrm{kcal} / \mathrm{semana} \\
\text { Mujer: }<270 \mathrm{kcal} / \mathrm{semana}\end{array}$ \\
\hline Agotamiento & $\begin{array}{l}\text { Cuestionario CES-D } \\
\text { Cuestionario de } \\
\text { energía }\end{array}$ & $\begin{array}{l}\text { Se hacen } 2 \text { preguntas: Qué tan } \\
\text { frecuente en la última semana } \\
\text { sintió que todo lo que hacía era un } \\
\text { esfuerzo?/como si no pudiera } \\
\text { continuar? (frecuente } \geq 3 \text { días, } \\
\text { poco frecuente } 0-2 \text { días). } \\
\text { Se hacen } 7 \text { preguntas sobre la falta } \\
\text { de energía durante el mes pasado. }\end{array}$ & $\begin{array}{l}\text { Positivo si responde frecuente a } \\
\text { cualquier pregunta. } \\
\text { Positivo si criterio mayor }+2 \text { de los } \\
6 \text { menores. }\end{array}$ \\
\hline Desgaste & $\begin{array}{l}\text { Pérdida de peso } \\
\text { Albúmina sérica }\end{array}$ & $\begin{array}{l}\text { Pérdida de peso relacionada con } \\
\text { dieta o ejercicio. } \\
\text { Medicion de albúmina sérica. }\end{array}$ & $\begin{array}{l}>10 \text { libras el año pasado } \\
<3.3 \mathrm{~g} / \mathrm{dl}\end{array}$ \\
\hline $\begin{array}{l}\text { Short Physical } \\
\text { Perfomance Battery }\end{array}$ & $\begin{array}{l}\text { Test de equilibrio } \\
\text { Test de la silla } \\
\text { Test de velocidad de } \\
\text { la marcha en } 5 \mathrm{~m}\end{array}$ & $\begin{array}{l}\text { Paciente en posición de semitandem } \\
\text { por } 10 \mathrm{~s} \text {; si es capaz solicitar que } \\
\text { permanezca en tamdem por } 10 \mathrm{~s} ; \text { si } \\
\text { no es capaz permanecer con los } \\
\text { pies uno al lado del otro por } 10 \mathrm{~s} \text {. } \\
\text { Paciente sentado en una silla con } \\
\text { espalda recta, pedir que se pare } 5 \\
\text { veces lo más rápido posible con las } \\
\text { manos cruzadas sobre el pecho. } \\
\text { Medir el tiempo que tarde en } \\
\text { levantarse las } 5 \text { veces. } \\
\text { Ya descrito. }\end{array}$ & $\begin{array}{l}0=\text { lado a lado } 0-9 \mathrm{~s} \\
1=\text { lado a lado } 10 \mathrm{~s} \\
2=\text { tándem } 0-2 \mathrm{~s} \\
3=\text { tándem } 3-9 \mathrm{~s} \\
4=\text { tándem } 10 \mathrm{~s} \\
0=\text { incapaz } \\
1=\geq 16,7 \mathrm{~s} \\
2=13,7-16,6 \mathrm{~s} . \\
3=11,2-13,6 \mathrm{~s} . \\
4=\leq 11,1 \mathrm{~s} \\
0=\text { incapaz } \\
1=>11,6 \mathrm{~s} . \\
2=8,3-11,5 \mathrm{~s} . \\
3=6,5-8,2 \mathrm{~s} . \\
4=\leq 6.4 \mathrm{~s} . \\
\text { Fragilidad score } \leq 5 / 12 .\end{array}$ \\
\hline Escala de Fried & $\begin{array}{l}\text { Velocidad de marcha } \\
\text { en } 5 \mathrm{~m} \\
\text { Prueba de fuerza de } \\
\text { tensión } \\
\text { Cuestionario de } \\
\text { actividad física } \\
\text { Cuestionario CESD } \\
\text { Pérdida de peso }\end{array}$ & Ya descrito. & \\
\hline
\end{tabular}

Adaptado de Afilalo, J et al. Frailty assessment in the cardiovascular care of older adults. JACC. 2014;63(8):747-762. 


\section{Conclusiones}

Los cambios fisiológicos de la vejez junto a las comorbilidades que se suman con los años, hacen que la población añosa tenga alto riesgo de sufrir el síndrome coronario agudo y sus complicaciones tanto agudas como crónicas. La evidencia científica ha demostrado que la revascularización quirúrgica o percutánea es superior a la terapia médica en estos pacientes. Sin embargo, se ha encontrado que el tratamiento que se les proporciona está lejos de ser el ideal, con pocos pacientes sometidos a estos procedimientos. Queda entonces no solo el compromiso del cuerpo médico de conocer la evidencia existente, si no llevarla a la práctica, con lo que muy probablemente se incidirá en mejores resultados y se lograran salvar más vidas y obtener mejor calidad de vida en el grupo de adultos mayores.

\section{Responsabilidades éticas}

Protección de personas y animales. Los autores declaran que para esta investigación no se han realizado experimentos en seres humanos ni en animales.

Confidencialidad de los datos. Los autores declaran que en este artículo no aparecen datos de pacientes.

Derecho a la privacidad y consentimiento informado. Los autores declaran que en este artículo no aparecen datos de pacientes.

\section{Conflicto de intereses}

Los autores de este artículo declaran no tener ningún conflicto de intereses.

\section{Bibliografía}

1. Alexander KP, Newby LK, Cannon CP, Armstrong PW, Gibler WB, Rich MW, et al. Acute coronary care in the elderly, part I: Non-ST-segment-elevation acute coronary syndromes: a scientific statement for healthcare professionals from the American Heart Association Council on Clinical Cardiology: in collaboration with the Society of Geriatric Cardiology. Circulation. 2007; 115:2549-69.

2. Dégano IR, Elosua R, Marrugat J. Epidemiology of acute coronary syndromes in Spain: estimation of the number of cases and trends from 2005 to 2049. Rev Esp Cardiol Engl Ed. 2013;66:472-81.

3. Jackson CF, Wenger NK. Enfermedad cardiovascular en el anciano. Rev Esp Cardiol. 2011;64:697-712.

4. McCune C, McKavanagh P, Menown IB. A Review of Current Diagnosis, Investigation, and Management of Acute Coronary Syndromes in Elderly Patients. Cardiol Ther. 2015;4:95-116.

5. Saunderson CED, Brogan RA, Simms AD, Sutton G, Batin PD, Gale CP. Acute coronary syndrome management in older adults: guidelines, temporal changes and challenges. Age Ageing. 2014;43:450-5.

6. Colombia. Ministerio de Salud y Protección Social, Colciencias, Universidad de Antioquia. Guía de práctica Clínica para el Síndrome Coronario Agudo. GPC-SCA. Bogotá, 2013.

7. Barywani SB, Li S, Lindh M, Ekelund J, Petzold M, Albertsson P, et al. Acute coronary syndrome in octogenarians: association between percutaneous coronary intervention and long-term mortality. Clin Interv Aging. 2015;10:1547-53.

8. Hanon O, Baixas C, Friocourt P, Carrié D, Emeriau J-P, Galinier $M$, et al. Consensus of the French Society of Gerontology and Geriatrics and the French Society of Cardiology for the management of coronary artery disease in older adults. Arch Cardiovasc Dis. 2009;102:829-45.

9. Cardiovascular Disease in the Elderly. En: Braunwald's Heart Disease: A Textbook of Cardiovascular Medicine. Tenth edition. Philadelphia: Elsevier; 2015. p. 1711-43.

10. Brieger D, Eagle KA, Goodman SG, Steg PG, Budaj A, White $\mathrm{K}$, et al. Acute coronary syndromes without chest pain, an underdiagnosed and undertreated high-risk group*: Insights from the global registry of acute coronary events. Chest. 2004;126:461-9.

11. O'Gara PT, Kushner FG, Ascheim DD, Casey DE, Chung MK, de Lemos JA, et al. 2013 ACCF/AHA guideline for the management of ST-elevation myocardial infarction: executive summary: a report of the American College of Cardiology Foundation/American Heart Association Task Force on Practice Guidelines: developed in collaboration with the American College of Emergency Physicians and Society for Cardiovascular Angiography and Interventions. Catheter Cardiovasc Interv Off J Soc Card Angiogr Interv. 2013;82:E1-27.

12. Amsterdam EA, Wenger NK, Brindis RG, Casey DE, Ganiats TG, Holmes DR, et al. 2014 AHA/ACC Guideline for the Management of Patients with Non-ST-Elevation Acute Coronary Syndromes: a report of the American College of Cardiology/American Heart Association Task Force on Practice Guidelines. J Am Coll Cardiol. 2014;64:e139-228.

13. Alexander KP, Roe MT, Chen AY, Lytle BL, Pollack CV, Foody JM, et al. Evolution in cardiovascular care for elderly patients with non-ST-segment elevation acute coronary syndromes: results from the CRUSADE National Quality Improvement Initiative. J Am Coll Cardiol. 2005;46:1479-87.

14. Gale CP, Cattle BA, Woolston A, Baxter PD, West TH, Simms $A D$, et al. Resolving inequalities in care? Reduced mortality in the elderly after acute coronary syndromes. The Myocardial Ischaemia National Audit Project 2003-2010. Eur Heart J. 2012;33:630-9.

15. Schoenenberger AW, Radovanovic D, Windecker S, Iglesias JF, Pedrazzini G, Stuck AE, et al. Temporal trends in the treatment and outcomes of elderly patients with acute coronary syndrome. Eur Heart J. 2016;37:1304-11.

16. De Luca G, Dirksen MT, Spaulding C, Kelbak H, Schalij M, Thuesen L, et al. Impact of Age on Long-Term Outcome After Primary Angioplasty With Bare-Metal or Drug-Eluting Stent (from the DESERT Cooperation). Am J Cardiol. 2013;112:181-6.

17. Savonitto S, Morici N, De Servi S. Treatment of Acute Coronary Syndromes in the Elderly and in Patients With Comorbidities. Rev Esp Cardiol Engl Ed. 2014;67:564-73.

18. Fox KAA, Clayton TC, Damman P, Pocock SJ, de Winter RJ, Tijssen JGP, et al. Long-Term Outcome of a Routine Versus Selective Invasive Strategy in Patients With Non-ST-Segment Elevation Acute Coronary Syndrome. J Am Coll Cardiol. 2010;55:2435-45.

19. Tegn N, Abdelnoor M, Aaberge L, Endresen K, Smith P, Aakhus $\mathrm{S}$, et al. Invasive versus conservative strategy in patients aged 80 years or older with non-ST-elevation myocardial infarction or unstable angina pectoris (After Eighty study): an open-label randomised controlled trial. Lancet. 2016.

20. Hlatky MA, Boothroyd DB, Bravata DM, Boersma E, Booth J, Brooks MM, et al. Coronary artery bypass surgery compared with percutaneous coronary interventions for multivessel disease: a collaborative analysis of individual patient data from ten randomised trials. Lancet. 2009;373:1190-7.

21. Dacey LJ, Likosky DS, Ryan TJ, Robb JF, Quinn RD, DeVries JT, et al. Long-term survival after surgery versus percutaneous intervention in octogenarians with multivessel coronary 
disease. Ann Thorac Surg. 2007;84(6):1904-11; discussion 190411.

22. Nicolini F, Contini GA, Fortuna D, Pacini D, Gabbieri D, Vignali L, et al. Coronary Artery Surgery Versus Percutaneous Coronary Intervention in Octogenarians: Long-Term Results. Ann Thorac Surg. 2015;99:567-74.

23. Zaman MJ, Stirling S, Shepstone L, Ryding A, Flather M, Bachmann $M$, et al. The association between older age and receipt of care and outcomes in patients with acute coronary syndromes: a cohort study of the Myocardial Ischaemia National Audit Project (MINAP). Eur Heart J. 2014;35:1551-8.

24. Afilalo J, Alexander KP, Mack MJ, Maurer MS, Green P, Allen LA, et al. Frailty assessment in the cardiovascular care of older adults. J Am Coll Cardiol. 2014;63:747-62.

25. Veerasamy M, Edwards R, Ford G, Kirkwood T, Newton J, Jones $D$, et al. Acute coronary syndrome among older patients: a review. Cardiol Rev. 2015;23:26-32.

26. Rowe R, lqbal J, Murali-Krishnan R, Sultan A, Orme R, Briffa N, et al. Role of frailty assessment in patients undergoing cardiac interventions. Open Heart. 2014;1:e000033.

27. Ekerstad N, Swahn E, Janzon M, Alfredsson J, Löfmark $\mathrm{R}$, Lindenberger $\mathrm{M}$, et al. Frailty is independently associated with short-term outcomes for elderly patients with non-ST-segment elevation myocardial infarction. Circulation. 2011;124:2397-404.

28. Grosmaitre P, Le Vavasseur O, Yachouh E, Courtial Y, Jacob $X$, Meyran S, et al. Significance of atypical symptoms for the diagnosis and management of myocardial infarction in elderly patients admitted to emergency departments. Arch Cardiovasc Dis. 2013;106:586-92.

29. Huber K, Bates ER, Valgimigli M, Wallentin L, Kristensen SD, Anderson JL, et al. Antiplatelet and anticoagulation agents in acute coronary syndromes: What is the current status and what does the future hold? Am Heart J. 2014;168:611-21.

30. Carroll KA, Early NK, Tsu LV. Managing acute coronary syndromes in the elderly. Consult Pharm J Am Soc Consult Pharm. 2015;30:265-82.

31. Antman EM, Morrow DA, McCabe CH, Murphy SA, Ruda M, Sadowski Z, et al. Enoxaparin versus unfractionated heparin with fibrinolysis for ST-elevation myocardial infarction. N Engl J Med. 2006;354:1477-88.

32. Sabatine MS, Cannon CP, Gibson CM, López-Sendón JL, Montalescot G, Theroux P, et al. Addition of clopidogrel to aspirin and fibrinolytic therapy for myocardial infarction with ST-segment elevation. N Engl J Med. 2005;352:1179-89.

33. Chen ZM, Jiang LX, Chen YP, Xie JX, Pan HC, Peto R, et al. Addition of clopidogrel to aspirin in 45,852 patients with acute myocardial infarction: randomised placebo-controlled trial. Lancet. 2005;366:1607-21.

34. Yusuf S, Zhao F, Mehta SR, Chrolavicius S, Tognoni G, Fox KK, et al. Effects of clopidogrel in addition to aspirin in patients with acute coronary syndromes without ST-segment elevation. N Engl J Med. 2001;345:494-502.

35. Yusuf S, Mehta SR, Zhao F, Gersh BJ, Commerford PJ, Blumenthal $\mathrm{M}$, et al. Early and late effects of clopidogrel in patients with acute coronary syndromes. Circulation. 2003;107:966-72.

36. Wiviott SD, Braunwald E, McCabe CH, Montalescot G, Ruzyllo W, Gottlieb S, et al. Prasugrel versus clopidogrel in patients with acute coronary syndromes. N Engl J Med. 2007;357:2001-15.

37. U.S. Food and Drug Administration FDA. Safety Information Effient (prasugrel hydrochloride) $5 \mathrm{mg}$ and $10 \mathrm{mg}$ Tablets [Internet] [consultado 6 Nov 2015]. Disponible en: http://www.fda. gov/Safety/MedWatch/Safetylnformation/ucm275490.htm

38. Raja SG. Myocardial revascularization for the elderly: current options, role of off-pump coronary artery bypass grafting and outcomes. Curr Cardiol Rev. 2012;8:26-36.

39. Graham MM, Ghali WA, Faris PD, Galbraith PD, Norris CM, Knudtson $\mathrm{ML}$, et al. Survival after coronary revascularization in the elderly. Circulation. 2002;105:2378-84.

40. Grines C, Patel A, Zijlstra F, Weaver WD, Granger C, Simes RJ, et al. Primary coronary angioplasty compared with intravenous thrombolytic therapy for acute myocardial infarction: six-month follow up and analysis of individual patient data from randomized trials. Am Heart J. 2003;145:47-57.

41. Forman DE, Chen AY, Wiviott SD, Wang TY, Magid DJ, Alexander KP. Comparison of Outcomes in Patients Aged \&lt;75, 75 to 84, and $\geq 85$ Years With ST-Elevation Myocardial Infarction (from the ACTION Registry-GWTG). Am J Cardiol. 2010;106:1382-8.

42. Alabas OA, Allan V, McLenachan JM, Feltbower R, Gale CP. Age-dependent improvements in survival after hospitalisation with acute myocardial infarction: an analysis of the Myocardial Ischemia National Audit Project (MINAP). Age Ageing. 2014;43:779-85.

43. Redfern J, Hyun K, Chew DP, Astley C, Chow C, Aliprandi-Costa $B$, et al. Prescription of secondary prevention medications, lifestyle advice, and referral to rehabilitation among acute coronary syndrome inpatients: results from a large prospective audit in Australia and New Zealand. Heart. 2014;100:1281-8.

44. Tegn N, Abdelnoor M, Aaberge L, Endresen K, Smith P, Aakhus $\mathrm{S}$, et al. Invasive versus conservative strategy in patients aged 80 years or older with non-ST-elevation myocardial infarction or unstable angina pectoris (After Eighty study): an open-label randomised controlled trial. The Lancet. 2016;387(10023):1057-65. 